\title{
THERMOECONOMIC OPTIMIZATION OF A HEAT PUMP SYSTEM
}

\author{
G. WAI.L. \\ Physical Resource Theory Group, Chalmers I niversity of Technology and l Iniversity of Göteborg. \\ S-41296 Göteborg. Sweden \\ (Receired 8 Norember 1985)
}

\begin{abstract}
We present the application of thermocconomics to the optimization of a single-stage heat-pump cycle. The method is well suited for application to thermodynamic processes and yields exergy losses. The marginal cost of an arbitrary variable can also be calculated. The efficiencies of the compressor, condenser, evaporator, and electric motor are chosen as the decision variables which are to be optimized. Parameters such as the price of electricity and the temperature of the delivered heat may vary among optimizations, and results are presented for different parameter values.
\end{abstract}

\section{INTRODUCTION}

Contemporary technical systems are often constructed without detailed prior analysis of the effects of different potential solutions for a given system in terms of cost. Instead, constructions are often based on experience, educated guesswork and personal evaluations. Sometimes energy reallocations are made, for example when the investment budget is based on calculation of energy (or, better, exergy) losses in each zone of the system. Irrespective of the method used, the maximum cost permitted for each zone is obtained, and the market prices then determine to what extent efficient components can be afforded. Such systems always cost at least as much as and often more than they would if thermoeconomic optimization were used.

Tribus and El-Sayed ${ }^{1.2}$ developed the concept of thermoeconomics. in which the objective function is optimized, subject to given economic and technical constraints. The purpose of thermoeconomics is to improve analyses of systems by introducing ways of concurrently suggesting improvements.

The result of an insufficient analysis is that we never know the best solution or how to find it. By optimizing the total system in operation, we always find the best system within the given conditions. We can also calculate the marginal costs of the exergy lusses in each component. These values are very important in the selection of research and development measures, or in the improvement of an existing system.

The system is described in relation to the physical (pressure $p_{0}$. temperature $T_{0}$, and chemical potentials of the appropriate substances $m_{c, 0}$ ) and economic cnvironments (prices of goods $c_{i}$ and prices of capital or interest rates $a_{i}$ ). These 2 environments are interrelated by cost relations for physical quantities.

Briefly, the method involves the following steps: (1) a concise description of the system under study; (2) definition of system boundaries, zones, components, etc. (a detailed flow chart or a draft of the process); (3) definitions of the physical environment or, alternatively. of the local physical environment; (4) declarations of the sources of the thermophysical data; (5) development of a computational algorithm with inputs and outputs clearly identified, based on mass and energy balances for the system and a complete thermodynamic description, within the given conditions; (6) definitions of the cost equations and the economic objective function; (7) determinations of the exergy flows and sources of entropy, i.e. exergy sinks, in the system (related to the in and outflows of exergy); (8) determination of the monetary flows in the process; (9) suggestions of possible improvements of the configuration of the system and adjustments of associated relations (5): (10) optimizations of the objective of the process, i.e. the cost; 111) carrying out of a sensitivity study; (12) suggested improvements of the design and areas for further research and development. 


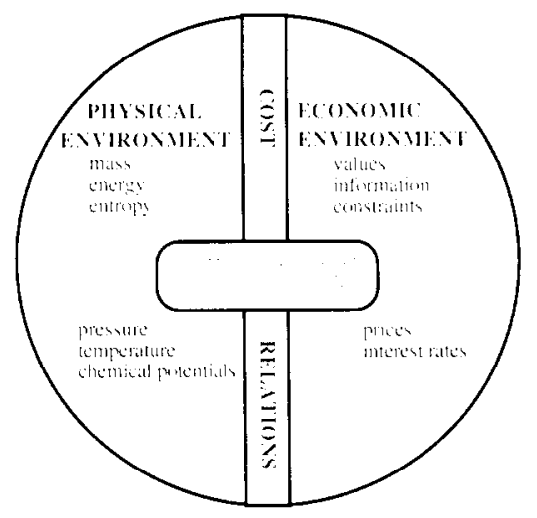

Fig. 1. The system in 2 environments.

The new element in this working scheme is introduction of the environment and its effects on the system. This makes it possible to use the exergy concept to calculate the amount of work lost due to irreversibilities in the system. Thermoeconomics find the optimum economic design within physical feasibility. All costs are based on life-time costs, which may also include maintenance costs, etc. We might not be able to realize this optimum solution for an existing system, but we can learn how to improve it. The method also provides new insights, which often lead to new, unexpected improvements. Interest in thermoeconomics from industrial management is increasing today. ${ }^{3}$

Thermodynamic systems can be completely described using equations of mass balance (for each substance), energy and entropy, in conjunction with thermophysical property relations and/or equations of state, equipment performance characteristics, thermokinetic or rate equations, and boundary/initial conditions. Given an adequate description of the process, it can be optimized using any current technique. The exergy flow gives a unique description of the potentials for improving efficiency.

The variables describing the system can usually be divided into basic state variables, variable decision variables, and fixed decision variables. ${ }^{2}$ These variables are all interrelated by the objective function and the equations of constraints. We might substitute the constraint equations into the objective function, leaving only the independent variables, i.e. the variable decision variables, to be optimized. However, this is not always mathematically desirable. Using Lagrange's method, we can maintain the fixed or dependent variables in the optimization. Their shadow, i.e. marginal, prices are then given by the Lagrange multipliers.

The application of Lagrange's method to large scale systems is well known and widespread. ${ }^{4}$ Methods are also available whereby a complex thermal system is decomposed into its component parts, each component buying and selling exergy with other components. ${ }^{5}$ However, this approach requires that the problem is described in terms of exergy coordinates, i.e. the constraint equations representing the internal economic transactions (supply and demand equations) must be explicitly given in terms of exergy flows. The benefit of such a transformation is that the Lagrange multipliers represent prices describing the internal sales and purchases of exergy. These prices can be used in turn to show the economic trade-offs between capital investment costs and exergy losses for each component of the system.

In order to obtain the exergy balance equations, all the relevant thermodynamic constraints must be incorporated. Usually there is no general way to find these constraint equations. All state variables (temperature, pressure, mass flows, etc.) must also be transformed into exergy variables, which may be problematical. Usually this transformation ends up in a number of non-linear equations which must be solved to get the optimum values for the decision variables. We avoid these problems by using numerical treatment.

When constructing a system, attempts should be made to attain the highest possible 
technical efficiency at the lowest cost within the existing technical, economical and legal constraints. (Sometimes we must also consider ethical, ecological or social consequences.) The analysis should also include different operating points (pressures, temperatures, etc.), configurations (components, flow charts, etc.), purposes (dual purpose, use of waste streams, etc.), and environments (global or local environment, new prices, etc.). As mentioned above, new areas for research and development should also be identified.

Computer programs and suitable strategies allow for examination of a large number of solutions, and determination of competitive solutions. A good strategy should include the use of new components and a suitable level of accuracy for available and assumed quantities representing different properties, performances and costs. The purpose of this study is to develop such a strategy from a fundamental theoretical basis and to formalize what many people regard as common sense. The point of departure is in regarding the system as surrounded by a physical and economic environment, see Fig. 1.

With regard to the physical environment, the energy and mass flows are evaluated in physical terms, i.c. in terms of exergy per unit time. The difference between all incoming exergy flows and all outgoing exergy flows must be minimized and the efficiency must be maximized. In the economic environment all energy and mass flows are evaluated instead in terms of economic value or costs. The main function is now the cost per unit time, (i.e. operation and capital costs minus income), which should be minimal. Thermoeconomic optimization is economic optimization in conjunction with thorough thermodynamic description of the system.

\section{EXERGY}

The exergy losses due to irreversibilities in a stationary state can be determined for each zone, a single component or a number of components, by regarding in and outflows of exergy. The exergy content $E$ of a flow is

$$
E=H-T_{0} S-\sum_{c} \mu_{c, 0} n_{c}
$$

where $H$ is the cnthalpy, $T_{0}$ the absolute tempcrature of the environment, $S$ the entropy, and $\mu_{c, 0}$ the chemical potential of substance $c$ of quantity $n_{c}$. The exergy loss can be determined for each zone. For the system, we obtain a sum for all zones, which gives the total rate of exergy loss. This may also be written as the product of the environment temperature and total rate of entropy production, i.e. $T_{0} \Delta S^{t o t}$.

\section{COSTS OF EXERGY LOSSES}

There are 2 main reasons for placing a price on exergy losses, irrespective of whether the purpose is physical or economic optimization. The first is to obtain a description of how the variable mass and energy flows in the objective function are distributed over the process and the second is to find a redistribution that fulfils the objectives. This corresponds to 2 kinds of analysis: thermoeconomic accounting with the use of direct prices which allows comparison between zones and their costs, disregarding other parts of the system, and thermocconomic optimization using differential prices, which allows comparison between the local and global responses of the system to a particular change of state. This leads to a sensitivity analysis and an optimization of the defined objective function.

The differential prices indicating the direction of improving the objective function are marginal prices and shadow prices. A marginal price indicates the change of the objective function at a unit change of a variable decision variable. The shadow price indicates the corresponding direction for the other variables. Marginal prices of exergy can then be calculated using the chain rule of derivation. 


\section{EFFICIENCY AND COST OPTIMIZATION}

The calculation of exergy flows and their costs requires a thermodynamic and economic description of the system. $\dagger$ A suitable level of description, when comparing alternative solutions, is the level at which efficiencies and costs for each subsystem are measured (as part of the system) in common quantities. A more detailed description can thus be made by dividing the subsystem into even smaller parts.

Important relations for describing the subsystems are equations for mass, energy, performances and costs as functions of performance. Cost relations as functions of capacity parameters such as areas, mass flows and powers are suitable in a preliminary analysis. ${ }^{6}$ These relations may later be rewritten to include other parameters such as temperatures, pressures or efficiencies.

\section{THERMOECONOMIC ACCOUNTING}

This method is one based on the exergy flows in the process, by which a price is put on the exergy. The exergy inflows are shared between useful outflows, wasted outflows and exergy losses. Thermoeconomic accounting simply means determining the exergy flows and assigning values to the exergy flows and losses. When there are various in and outflows the prices may vary. If the price per exergy unit does not vary too greatly we can define an "average price". This method allows comparison of the economic cost of a zone with the cost of its exergy loss.

Thermoeconomic accounting does not include consideration of the system effects. It does not describe how the capital investments in one part on the system affect cxcrgy losses in other parts of the system. In the thermoeconomic accounting method the exergy losses are only figures not functions. However, this simple type of analysis sometimes gives ideas for unusual improvements.

\section{THERMOECONOMIC OPTIMIZATION}

Direct prices are not affected when a change in one part of the system affects other parts. Marginal and shadow prices are affected, but they are difficult to determine. According to the system equations they are dependent on the first derivatives of the parameters in question and not only on their absolute values.

The objective function $\Phi_{0}$ must be defined as a function of dependent state variables or state paramctcrs $\left\{x_{j}\right\}, \ddagger$ variable decision variables or just decision variables $\left\{y_{k}\right\}$, and fixed decision variables or decision parameters $\left\{z_{l}\right\}$, i.e.

$$
\Phi_{0}=\Phi_{0}\left(\left\{x_{j}\right\},\left\{y_{k}\right\},\left\{z_{l}\right\}\right)
$$

where $j=1,2, \ldots, n, k=1,2, \ldots, m$, and $l=1,2, \ldots, r$.

The equations of state may be divided into 2 groups. One group fixes the dependent variables $\left\{x_{j}\right\}$, appearing in the objective function $\Phi_{0}$ and the constraints, $\left\{\Phi_{j}=0\right\}$, in Lagrange's method. The other group is related to the Lagrange multipliers $\left\{\lambda_{j}\right\}$. The equations of state are written as

$$
\Phi_{j}=\bar{\Phi}_{j}-x_{j}
$$

where $\Phi_{j}$ is the function used in Lagrange's method since it must be zero at optimum, i.e. when $x_{j}=\bar{\Phi}_{j}, \bar{\Phi}_{j}$ gives the relations between the state variable in question $x_{j}$ and the other

† Second law considerations are not needed in the optimization. The exergy concept is only used to discover possible future improvements beyond the economic improvements.

$\ddagger$ Here, we let $x_{j}$ denote the $j$ th state parameter and write $\left\{x_{j}\right\}$ in the abbreviated forms $x_{1}, x_{2}, \ldots, x_{j}, \ldots, x_{n}$. 
state variables $\left\{x_{i}\right\}_{i \neq j}$ and the decision variables $\left\{y_{k}\right\}$ and $\left\{z_{l}\right\}$. With these definitions, the equations of state become

$$
\Phi_{j}\left(\left\{x_{i}\right\},\left\{y_{k}\right\},\left\{z_{l}\right\}\right)=0, \quad j=1,2, \ldots, n
$$

The problem can now be formulated as follows:

$$
\begin{gathered}
\operatorname{minimize} \Phi_{0}=\Phi_{0}\left(\left\{x_{i}\right\},\left\{y_{k}\right\},\left\{z_{l}\right\}\right), \\
\text { subject to } \Phi_{j}\left(\left\{x_{i}\right\},\left\{y_{k}\right\},\left\{z_{l}\right\}\right)=0, \quad j=1,2, \ldots, n .
\end{gathered}
$$

With the defined objective function and equations of state, the Lagrangian becomes

$$
L=\Phi_{0}+\sum_{j} i_{j} \Phi_{j}
$$

According to Lagrange's method,

$$
\partial L / \partial x_{i}=0, \quad i=1,2, \ldots, n
$$

This procedure gives $n$ equations, which are linearly dependent through the unknown Lagrange multipliers $\left\{\lambda_{j}\right\}$, which may be regarded as shadow prices. ${ }^{7}$

When the objective function and the equations of state are not too complicated, the optimum is given by

$$
\partial L / \partial y_{k}=0, \quad k=1,2, \ldots, m
$$

The optimum solution is found by solving these equations for the variable decision variables $\left\{y_{k}\right\}$.

In real world applications, it is often better to define a marginal price $\theta k$, related to each variable decision variable $y_{k}$, i.e.

$$
\theta_{k}=\frac{\partial L}{\partial y_{k}}=\frac{\partial \Phi_{0}}{\partial y_{k}}+\sum_{i=1}^{n} \lambda_{i} \frac{\partial \Phi_{i}}{\partial y_{k}}, \quad k=1,2, \ldots, m
$$

The physical interpretation of these prices is that they constitute the gradients for each variable decision variable, $y_{k}$, i.e. they give the direction for improving the system.

Equation (8) represents $n$ linear equations for the unknown Lagrange multipliers and Eq. (9) represents $m$ equations with the variable decision variables as unknowns. In many real world applications of this method the $n+m$ equations defined by Eqs (8) and (9) are strongly non-linear and difficult to solve. Numerical treatment is therefore often necessary. Numerical values must then be calculated for the shadow prices $\left\{\lambda_{j}\right\}$ for every set of the variable decision variables $\left\{y_{k}\right\}$. These shadow prices are then inserted into Eq. (10) to determine the marginal prices $\left\{\theta_{k}\right\}$. The marginal prices represent the derivatives of the objective function $\Phi_{0}$ in the state $i$. From the values of the marginal prices we can now indicate the direction for improving the system. A new state, $i+1$, is chosen in this direction according to

$$
y_{k, i+1}=y_{k, i} \pm \Delta y_{k}
$$

where $\Delta y_{k}$ is a predefined step.

When the marginal price $\theta_{k, i}$ is negative then the derivative of the objective function is 
negative. If we want to minimize the objective function, we must increase the variable decision variable $y_{k, i}$ to reach the optimum. If the marginal price is positive we must decrease the variable decision variable.

\section{SYSTEM MODEL}

Heat pump systems offer much more efficient means of producing heat than traditional combustion or electrical short circuit technologies. Heat pump systems are therefore becoming more common as the prices of fuels and electricity increase. The configuration of the system is defined in Fig. 2. It consists of a compressor, a condenser, an expansion valve, an evaporator, and an electric motor.

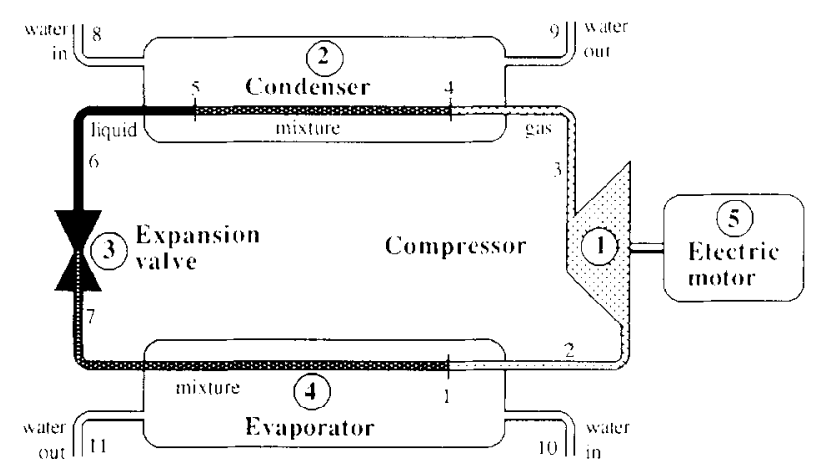

Fig. 2. Heat pump system with 5 components and 11 flows.

The refrigerant is superheated after passing through the evaporator, step $1-2$, and supercooled after passing through the condenser, step 5-6. The actual state of the refrigerant after the compression, 3 , differs from that of a reversible process, $3_{r e v}$, due to the limited efficiency of the compressor. The heat produced from the system is $h_{3}-h_{6}$, the heat input is $h_{2}-h_{7}$, and the work supplied to the compressor is $h_{3}-h_{2}$. The electricity input required to operate the system becomes $\left(h_{3}-h_{2}\right) / \eta_{5}$, where $\eta_{5}$ is the efficiency of the electric motor.

Variable decision variables are the efficiencies of the compressor, the condenser, the evaporator, and the electric motor. These are defined as follows

$$
\begin{gathered}
\eta_{1}=\left(h_{3, \text { rev }}-h_{2}\right) /\left(h_{3}-h_{2}\right), \\
\eta_{2}=\left(T_{9}-T_{8}\right) /\left(T_{4}-T_{8}\right), \\
\eta_{4}=\left(T_{11}-T_{10}\right) /\left(T_{1}-T_{10}\right) \\
\eta_{5}=m_{r}\left(h_{3}-h_{2}\right) / P
\end{gathered}
$$

where $m_{r}$ is the mass flow of the refrigerant, and $P$ is the electric power.

The system is completely defined apart from the variable decision variagbles $\left\{y_{k}\right\}$, each set of which determines a state of the system. The exergy flows and exergy losses are also determined for each component.

The objective is to minimize the cost for a given amount of produced heat. The cost includes both the operating (electricity) cost and the capital cost. The operating cost increases if the investments decrease and vice versa. The income from the product (heat) and a given required value of the profit sets an upper limit for the total cost of the system. The problem is to split this cost between the operating cost and the capital cost for each component. (The costs for parts not affected by alternative constructions of the system, 
such as pipes connecting the components, are just added as constants and have no effect on the optimization.) The costs, $C$, for each of the 5 components will now be given.

Compressor

$$
C_{1}=a_{1} k_{1}\left[V_{2} /\left(0.9-\eta_{1}\right)\right]\left(p_{3} / p_{2}\right)\left[\ln \left(p_{3} / p_{2}\right)\right]
$$

where $k_{1}$ is the cost per volume flow, $V_{2}, p_{3} / p_{2}$ is the pressure ratio over the compressor, and $\eta_{1}$ is the enthalpy efficiency.

Condenser $\quad C_{2}-a_{2} k_{2} m_{w h} \sqrt{\eta_{2} /\left(1-\eta_{2}\right)}=a_{2} k_{2} m_{w h} \sqrt{\mathrm{e}^{N T U_{2}}-1}$,

where $k_{2}$ is the cost per mass flow of water, $m_{w h}$, on the hot side; $\eta_{2}$ is the temperature efficiency, and $\mathrm{NTU}_{2}$ is the number of heat transfer units.

Expansion Value $\quad C_{3}=a_{3} k_{3} m_{\mathrm{r}}$,

where $k_{3}$ is the cost per mass flow of the refrigerant.

Evaporator $\quad C_{4}=a_{4} k_{4} m_{w c} \sqrt{\eta_{4} /\left(1-\eta_{4}\right)}=a_{4} k_{4} m_{w c} \sqrt{\mathrm{e}^{\mathrm{NT} U_{4}}-1}$,

where $k_{4}, \eta_{4}$ and $\mathrm{NTU}_{4}$ are anlogous to the condenser above and $m_{w r}$ is the mass flow of water on the cold side.

Electric Motor $\quad C_{5}=a_{5} k_{5} P \eta_{5}\left(1-\eta_{5}\right)$,

where $k_{5}$ is the cost per unit power and $\eta_{5}$ is the efficiency. Furthermore $a_{1}-a_{5}$ are annuity factors of the different capital investments defined as

$$
a_{i}=r\left[1-(1+r)^{-n_{i}}\right],
$$

where $r$ is the interest rate and $n_{i}$ is the depreciation time for component $i$. The depreciation time may vary for each component due to variations in economic lifetime and maintenance costs such as renovations, etc.

Figure 3 shows the investment costs as a function of the efficiencies. The values of the fixed decision variables are set, for intance as follows: heat produced $6500 \mathrm{~W}$ (energypower), operation time $/ \mathrm{yr} 5000 \mathrm{hr}$, price of electricity $0.25 \mathrm{SEK} / \mathrm{kWh}$, and temperature of the produced heat $\left(T_{9}\right) 60^{\circ} \mathrm{C}$.

The investment costs are depreciated according to the annuity method, which gives a cost per unit time for every component. The total cost per unit time $\Phi_{0}(=$ objective function) is the sum of these costs and the cost of the electricity used, $E_{c l}$, i.e.

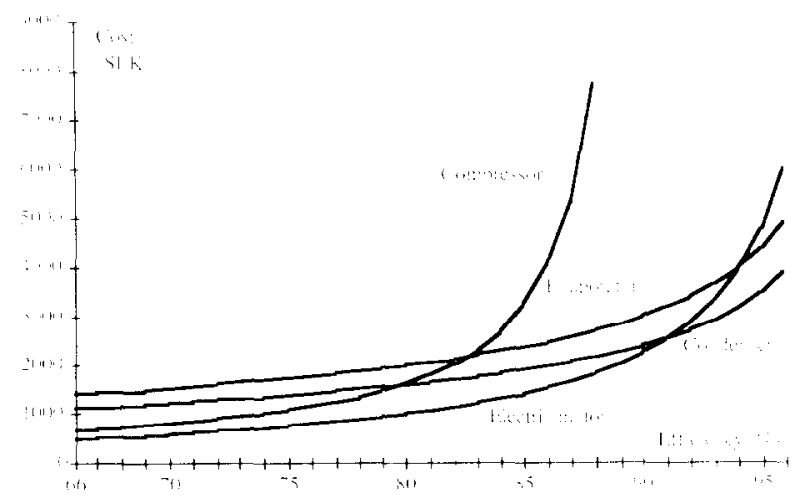

Fig. 3. Costs of investments as a function of the efficiencies. 


$$
\Phi_{0}=\sum_{i=1}^{5} C_{i}+t p_{e l} E_{e l}
$$

where $t$ is the operating time per unit time and $p_{e l}$ is the price of electricity.

This optimization problem is too complicated to be solved by Lagrange's method. Instead, the optimization is achieved by calculating the value of the objective function $\Phi_{0}$ and the marginal costs $\left\{\theta_{k}\right\}$ for every set of the variable decision variables $\left\{y_{k}\right\}$ according to

$$
\theta_{k}=\Delta \Phi_{0} / \Delta y_{k}, \quad k=1,2, \ldots, 4 .
$$

From these values a new set of variable decision variables $\left\{y_{k}\right\}$ is determined by using the Newton-Raphson method. ${ }^{8}$ Thus the system moves towards the nearest minimum from the given start values. However, the problem is strongly non-linear which means that there is no general method for finding the global minimum. Instead common sense and insight into how the system works must be used to determine the value of a solution.

A computer program has been developed for finding the optimum system. The program begins by calculating the thermodynamic data for the assumed refrigerant R12. (Other refrigerants may also be used.) These calculations are based on similar computer-based calculations by Reynolds. ${ }^{9}$ The equations of state for the system are formulated so as to avoid iterations. The actual minimizing procedure is carried out with a small number of iterations. When the sum of the marginal prices is less than a predefined value the optimization is completed.

Let us assume a system with the value $0.7 \dagger$ for all efficiencies, the variable decision variables, which are to be optimized. The calculated total cost then becomes SEK 4221/yr of which SEK $3617 / \mathrm{yr}$ relate to electricity. The optimization gives the following efficiency values: compressor 0.80 , condenser 0.83 , evaporator 0.73 and electric motor 0.91 , the total cost now amounting to SEK 3388/yr of which SEK 2416/yr is for the electricity. By increasing the investments from SEK 604/yr to SEK 972/yr, the total cost of the system becomes SEK 833/yr less than for the assumed system, see Fig. 4. At the same time the exergy losses decrease from $1933 \mathrm{~W}$ to $979 \mathrm{~W}$, i.e. $954 \mathrm{~W}$ ! From Fig. 5 we see that it the improvement of the electric motor that gives the largest single exergy saving. The optimization saves both costs and exergy.

The expansion valve accounts for the largest exergy loss in the optimum system, which justifies investment in research and development to improve it. ${ }^{10}$

The result indicates the electric motor to be the most critical component to improve. The electric motor is assumed to cost approximately 3 times as much at $91 \%$ efficiency than at $70 \%$ efficiency, which must be regarded as realistic. (It may even cost up to 9 times as much and still be competitive with the assumed system.)

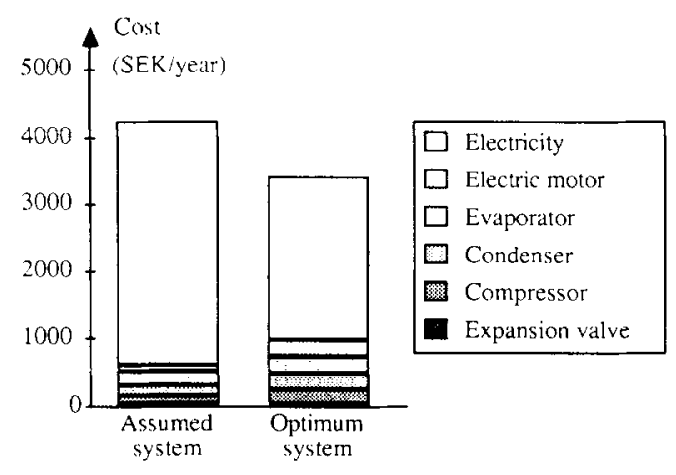

Fig. 4. Costs for the assumed and the optimum systems. 


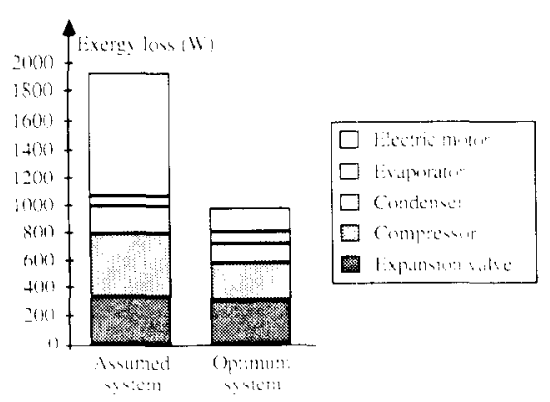

Fig. 5. Exergy losses for the assumed and the optimum systems.

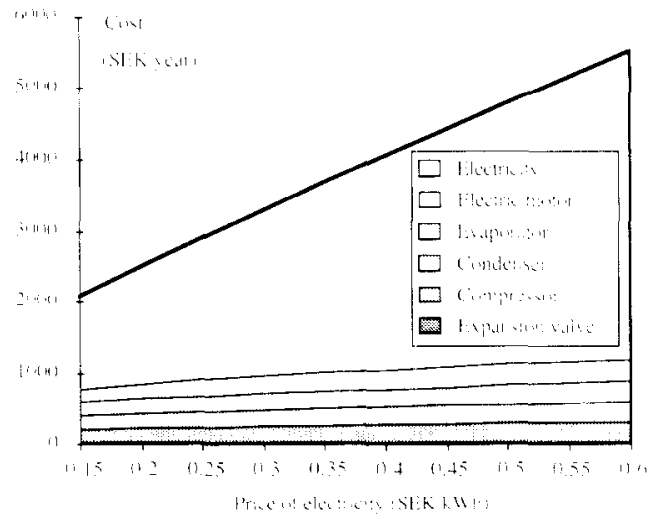

Fig. 6. Costs as a function of the price of electricity.

The dependence on or sensitivity to the fixed decision variables (price of electricity and temperature of the produced heat) has also been studied. Figure 6 shows the relationship between the costs and the price of electricity, when this varies between SEK 0.15 and $0.6 / \mathrm{kWh}$. The total cost then increases from SEK $2073 / \mathrm{yr}$ to SEK $5522 / \mathrm{yr}$ at SEK $0.6 / \mathrm{kWh}$. This can be seen in relation to the fact that if the optimum system at SEK $0.15 / \mathrm{kWh}$ had been used at SEK 0.6/kWh, then the total cost would be SEK 5947, i.e. an increase in the cost of SEK 425/yr. (For the assumed system the total cost would instead by SEK 9284, i.e. a cost increase of SEK 3762/yr.) Figure 7 shows more clearly the relationship between component costs and the price of electricity. All components should become more efficient (and therefore more expensive) when the price of electricity increases. This might have been anticipated, but the exact inter-relations could not have been hypothesized.

When the temperature of the condenser, i.e. the temperature of the produced heat, is changed interesting things occur (Figs 8 and 9). The total cost doubles from 2336 at $40^{\circ} \mathrm{C}$

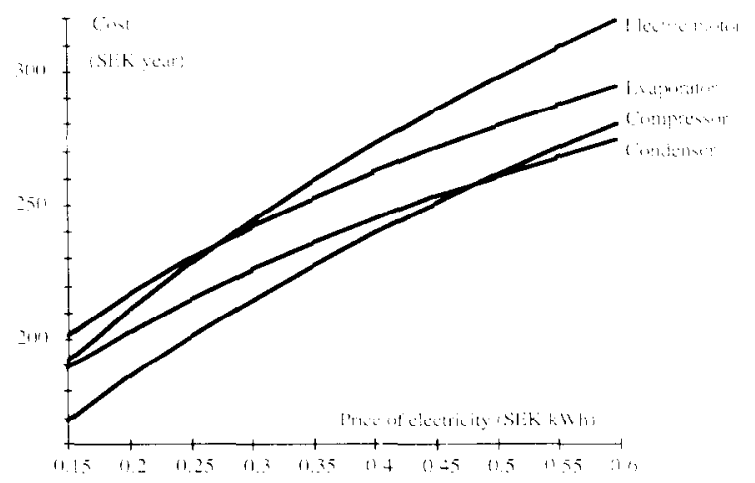

Fig. 7. Component costs as a function of the price of electricity. 
to SEK $4680 / \mathrm{yr}$ at $75^{\circ} \mathrm{C}$. The heat produced in energy per time units is the same, i.e. $6500 \mathrm{~W}$, but in exergy per time units it changes from 434 to $713 \mathrm{~W}$ which better explains the increased cost. When the temperature increases from 40 to $75^{\circ} \mathrm{C}$ the total system, but not necessarily each component, must be more efficient, see Fig. 9. Within a total increase of component costs and efficiencies, it is more economical to choose a less expensive evaporator. The explanation is simply that investments in other parts of the system pay off better.

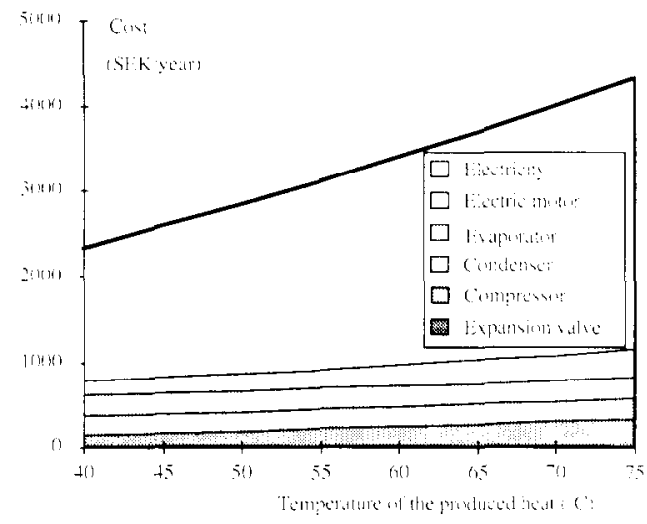

Fig. 8. Costs as a function of the temperature of the produced heat.

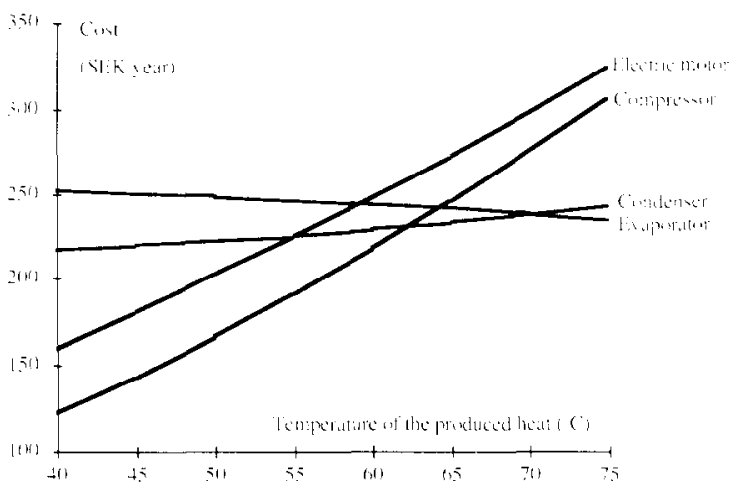

Fig. 9. Component costs as a function of the temperature of the produced heat.

Similarly, all other relations may be described using the computer program for the system. The program can easily be rewritten for other refrigerants or cost relations. The purpose of this study is merely to show the gain achieved by applying the method of thermoeconomics to a heat pump process. The exact numerical results for describing thermoeconomics as a method for improving technical processes have therefore been neglected.

Thermoeconomics can never replace long experience and high technical and economic competence, but it might be an important complementary tool.

Acknowledgements-This work was supported by an Operating Grant from the National Swedish Board for Technical Development, for which support the writer is most grateful. I thank M. Palmblad for helping me with the computer program and reading the manuscript at a very preliminary stage. I also thank $\mathrm{K}$. E. Eriksson and S. Karlsson for reading the manuscript.

Note - All computer programs are available upon request. 


\section{REFERENCES}

1. Y. M. El-Sayed and M. Tribus. A Specific Strategy for the Improtement of Process Economics. Center for Advanced Engineering Study, M.I.T., Cambridge, Mass., U.S.A. (1982).

2. Y. M. El-Sayed and M. Tribus. Strategic use of thermoeconomics for systems improvement. Efficiency und Costing. (Edited by R. A. Gaggioli), ACS Symposium series No. 235 (1983).

3. A. Reis, I. Smith, K. Stephan and J. L. Peube. Energy cconomics and management in industry. Proc. Fur. Cong., Algarve, Portugal, 2. 5 April 1984, Pergamon Press (1985)

4. R. M. Garceau and W. J. Wepfer. Thermoeconomic optimization of a rankine cycle cogeneration system. Efficiency and Costing. (Edited by R. A. Gaggioli). ACS Symposium series No. 235 (1983).

5. Y. M. Ei-Sayed and R. B. Evans. J. Engng Power 27, (January. 1970).

6. K. K. Humphreys and S. Katell. Basic Cost Engineering. Marcel Dekker, New York (1981).

7. Y. M. El-Sayed and M. Tribus. The strategic use of thermoeconomic analysis for process improvement Presented at the A.I.Ch.E. Meeting, Detroit (August, 1981).

8. R. L. Johnston. Numerical Methods: a Software Approach. Wiley, New York (1982).

9. W. C. Reynolds. Thermodynamic properties in SI Graphs. Tables and Computational Equations for 40 Substances. Department of Mechanical Engineering, Stanford Univ.. Calif., U.S.A. (1979).

10. F: Granryd. Research project no 80-4062 supported by the National Swedish Board for Technical Development. Box 432 00, S-100 72 Stockholm. Sweden (1985). 